\title{
Phylogenetic relationships in Cortinarius with focus on North European species
}

\author{
ØYVIND STENSRUD, RUSSELL J.S. ORR, KJETIL REIER-RØBERG, \\ TROND SCHUMACHER and KLAUS HØILAND
}

STENSRUD, Ø., ORR, R.J.S., REIER-RØBERG, K., SCHUMACHER, T. \& HØILAND, K. 2014: Phylogenetic relationships in Cortinarius with focus on North European species. - Karstenia 54: 57-71. HELSINKI. ISSN 0453-3402.

\begin{abstract}
Cortinarius is an ectomycorrhizal Agaricales genus with high diversity of which rDNA sequences of 86 species together with four outgroup taxa were investigated phylogenetically by aid of Maximum Likelihood and Bayesian analyses. The Cortinarius data set represents 81 taxa from the Northern Hemisphere showing the main variation spectrum among the species. In addition, five species from the Southern Hemisphere are included. The phylogenetic tree of Cortinarius gives statistical support to twelve monophyletic groups in the upper level. They are discussed in context of morphology, chemistry (secondary compounds), and ecology. The phylogenetic tree lacks, however, satisfactory support for its backbone. Several species could not be included in any group, especially those forming the basal framework of the tree. Of special interest is a "superclade" comprising eight of our monophyletic clades and two singletons. Here we find the majority of species with soluble pigments of octaketide origin, all species with compounds of nonaketide origin, the majority of species with hygrophaneous pileus, few species with viscid pileus, and no species with bulbous stipe base. Moreover, all species except one have duplex pileus cuticle. The morphological traits are not indicative for any clade, although some are more frequent in some clades than others. During the evolution they have been gained and lost several times. The chemical characteristics are - to a certain degree - more indicative for the clades. The evolution and ecological role of these compounds are discussed. Concerning the North European species, there are ecological differences between the clades, especially between clades specializing to rich or calcareous forests and clades specializing to poor forests or arctic-alpine environments.
\end{abstract}

Key words: Cortinarius, phylogeny, morphology, secondary compounds, ecology

Øyvind Stensrud \& Russell J.S. Orr \& Trond Schumacher \& Klaus Høiland (Corresponding author; e-mail: klaus.hoiland@ibv.uio.no), Department of Biosciences, University of Oslo, PO Box 1066 Blindern, NO-0316 Oslo, Norway

Kjetil Reier-Røberg, Department of mathematics and science, Buskerud and Vestfold University College, PO Box 4, NO-3199 Borre, Norway

\section{Introduction}

Cortinarius (Pers.) Gray is a genus of Agaricales (Basidiomycota) characterised by a fugacious veil enveloping the basidiocarp, and a cortina at first covering the lamellae, but later vanishing in expanding basidiocarps. The basidiocarp size, shape, and colour vary considerably among species. The lamellae are emarginate to adnate, variously coloured, soon becoming brownish from mature, brown-pigmented basidiospores. The basidiospores are usually verrucose, highly variable 
in size, and the colour may vary from pale ochre fulvous to rusty brown.

Diversity within the Cortinarius is high; over 2000 species are referred to the genus (Brandrud et al. 1990-2013). Cortinarius spp. are important ectomycorrhiza formers (Kårén et al. 1996) in temperate deciduous and boreal coniferous forests and arctic and alpine tundra (Ammirati \& Smith 1978, Moser 1983, Gulden \& Torkelsen 1996, Brandrud et al. 1990-2013) but do occur in tropical areas (Peintner et al. 2003) or Mediterranean evergreen thickets (Suáres-Santiago et al. 2009). The genus is also common and widespread in the Southern hemisphere (Moser \& Horak 1975, Keller et al. 1988, Chambers et al. 1999, Garnica et al. 2003a, Soop 2013, Stefani et al. 2014). The genus Cortinarius is inferred to be originally associated with angiosperms with a probable origin in late Cretaceous (Ryberg \& Matheny 2011).

Secondary compunds in the genus are frequent in many species, often characterising whole groups. Cortinarius orellanus Fr. and C. rubellus Cooke contain the highly nephrotoxic bipyridine orellanine (Schumacher \& Høiland 1983, Danel et al. 2001). Interesting alkaloids or (R)- $\beta$-dopa derivatives are found in $C$. infractus (Pers.) Fr., $C$. subtortus (Pers.) Fr., and C. violaceus (L.) Gray (von Nussbaum et al. 1998, Spiteller et al. 2000, Brondz et al. 2007, Brondz \& Høiland 2008, Teichert et al. 2008). All species of the infrageneric group Dermocybe (Fr.) Trog and some species belonging to the groups Telamonia (Fr.) Trog. and Phlegmacium (Fr.) Trog (the latter in the traditional circumscription (Brandrud et al. 1990-2013)) contain vividly coloured anthraquinones or preanthraquinones belonging to the octaketides (Gill $\&$ Steglich 1987). Cortinarius venetus (Fr.) Fr., $C$. cotoneus Fr., and C. colymbadinus Fr. contain yellow-green fluorescent xanthones and anthraquinones belonging to the nonaketides or octaketides (Gill \& Steglich 1987).

Traditionally Cortinarius has been divided into several infrageneric groups according to various authors (see Garnica et al. (2005) for a nice overview). The traditional subgenera included in this paper follow the treatment by Moser (1983) and Moser \& Horak (1975): Cortinarius, Dermocybe (by us at subgenus level), Leprocybe M.M. Moser., Myxacium (Fr.) Trog, Phlegmacium, Sericeocybe P.D. Orton, and Telamonia. The aforementioned traditional classifications are only partly support- ed by molecular phylogenetic studies (Høiland \& Holst-Jensen 2000, Peintner et al. 2003, Peintner et al. 2004, Garnica et al. 2005, Harrower et al. 2011, Ryberg \& Matheny 2011). Cortinarius violaceus, the type species of the genus (Clements \& Shear 1931), belongs to subgenus Cortinarius in the traditional classification. This subgenus has only ten global species (Peintner et al. 2004) and is characterised by basidiocarps with dry, squamulose pileus surface, lamellae with cheilo- and pleurocystidia, and spores with a distinct suprahilar smooth spot, a plage. The peculiar violet iron complex is unique for $C$. violaceus (von Nussbaum et al. 1998, Spiteller et al. 2000).

The monophyly of Telamonia is supported by several authors (Liu et al. 1995, Chambers et al. 1999, Høiland \& Holst-Jensen 2000, Peintner et al. 2003, Peintner et al. 2004, Garnica et al. 2005, Harrower et al. 2011), only Acetosi, Camphorati, Fulvescentes, Illumini, Laeti, Obtusi, and Renidentes are not included based on molecular studies (Niskanen 2008). The name Telamonia is vindicated since the clade includes the type species, C. torvus (Fr.) Fr. (Singer \& Smith 1946), of this subgenus (Garnica et al. 2005). The traditional subgenus Sericeocybe (Orton 1958) is, however, engulfed in clade Telamonia (Peintner et al. 2004, Garnica et al. 2005), since it contains the type species of the former: C. alboviolaceus (Pers.) Fr. (Singer 1986). A sister relationship between $C$. brunneus (Pers.) Fr. and C. gentilis (Fr.) Fr. has previously been documented (Høiland \& Holst-Jensen 2000, Niskanen et al. 2009), as well as between $C$. alboviolaceus and $C$. armeniacus (Schaeff.: Fr.) Fr. (Peintner et al. 2001).

Dermocybe s. str., (Høiland 1984), including the type, C. cinnamomeus (L.) Fr. (Singer 1986), is well supported (Liu et al. 1995, 1997; Chambers et al. 1999; Høiland \& Holst-Jensen 2000; Peintner et al. 2004; Garnica et al. 2005; Harrower et al. 2011). Common for all species are pigments of octaketide origin (Høiland 1984, Gill \& Steglich 1987). Two monophyletic lineages are identified in European and North American material, one Dermocybe line and one Sanguinei line, where C. malicorius Fr. occupies a basal position (Niskanen et al. 2012, 2013b). However, the Southern Hemispere species previously put in Dermocybe (Moser \& Horak 1975) form clades outside this group: Splendidi (with C. splendidus (E. Horak) K. Griffiths and C. kula Grgurinovic) forms an own clade; Icteri- 
nula (with C. icterinus (E. Horak) E. Horak and $C$. amoenus (M.M. Moser \& E. Horak) G. Garnier) form a third entity; and Pauperae (with C. luteostriatulus (M.M. Moser \& E. Horak) E. Valenz. \& G. Moreno, C. obscuroolivaceus (M.M. Moser) Kuhn.-Fink. \& Peintner, and C. flavifucatus (E. Horak \& M.M. Moser) E. Valenz \& G. Moreno), a fourth group (Garnica et al. 2003a, 2005; Peintner et al. 2004; Stefani et al. 2014).

The thin fleshed hygrophaneous, Telamonialike C. obtusus (Fr.) Fr. and C. acutus (Pers.) Fr. have repeatedly been demonstrated as a clade, $\mathrm{Ob}$ tusi, outside Telamonia (Høiland \& Holst-Jensen 2000, Peintner et al. 2003, 2004; Garnica et al. 2005; Harrower et al. 2011; Niskanen 2008).

Clade Leprocybe comprises species with strongly fluorescent nonaketide xanthones (Gill \& Steglich 1987). This clade represents the remains of subgenus Leprocybe (Peintner et al. 2004, Harrower et al. 2011), since it contains C. cotoneus, the type species (Singer 1986).

Clade Orellani comprises the toxigenic $C$. orellanus and C. rubellus. Biosynthesis of the bipyridines orellanine and orelline (Schumacher \& Høiland 1983, Gill \& Steglich 1987) is a monophyletic trait. Orellani was originally described as a section of subgenus Leprocybe (Moser 1969), but later elevated to a subgenus of its own (Gasparini 2004).

The subgenus Myxacium, as traditionally treated, has been divided into four sections: Myxacium, Defibulati, Delibuti, and Vibratiles/Ochroleuci (Høiland \& Holst-Jensen 2000, (Seidl 2000, Peintner et al. 2004, Garnica et al. 2005, Harrower et al. 2011). Section Myxacium comprises the core of this subgenus, including the type species, C. collinitus (Pers.) Fr. (Singer 1986), with Defibulati as sister clade. The two other sections are not related to these clades, and subgenus Myxacium with its original inventory is polyphyletic.

Subgenus Phlegmacium in its traditional sense appears para- or polyphyletic in molecular phylogenies (Høiland \& Holst-Jensen 2000; Garnica et al. 2003b, 2005, 2009); Peintner et al. 2004). Calochroi forms a distinct and strongly supported clade and comprises species with stout basidiocarps, simple cutis, and emarginated bulb at stipe base (Garnica et al. 2003b, 2005; Peintner et al. 2004; Frøslev et al. 2005, 2007); (Harrower et al. 2011). Scauri/Purpurascentes includes species with violet-red reaction in $\mathrm{KI}_{3}$ (Høiland \&
Holst-Jensen 2000; Garnica et al. 2003b, 2005; Harrower et al. 2011). The aforementioned studies also reveal several smaller, isolated clades. The remaining part of the traditional subgenus Phlegmacium constitutes a weakly supported group, comprising several evolutionary lineages (Garnica et al. 2005), e.g. Caerulescentes, Phlegmacioides, Percomes, Phlegmacium, containing the type species C. saginus (Fr.) Fr. (Singer 1986), and Praestantes.

The genus Rozites P. Karst. is characterised by having membranaceous partial veil forming a persistent ring and a membranaceous universal veil. It has previously been shown that Rozites should be included within Cortinarius (Høiland \& HolstJensen 2000, Peintner et al. 2002, Garnica et al. 2005, Harrower et al. 2011). It has been demonstrated that Rozites is polyphyletic, suggesting that a membranaceous veil has evolved in multiple independent events in Cortinarius (Peintner et al. 2002). Some fungi with sequestrate (gastroid or partially closed) basidiocarps, have a polyphyletic origin within genus Cortinarius (Peintner et al. 2001, Danks et al. 2010).

The aim of this study was to (1) evaluate the evolutionary relationship in a molecular data set of Cortinarius, including representative species for the different subgenera and sections, performing phylogenetic analyses of the ribosomal gene locus, containing the $18 \mathrm{~S}, 5.8 \mathrm{~S}$, and $28 \mathrm{~S}$ rDNA genes and the internal transcribed spacer regions (ITS1 and ITS2), and (2) trace infrageneric evolution of macro- and micromorphological, chemical, and ecological characteristics in light of the inferred molecular phylogeny, with focus on the North European species.

\section{Material and methods}

\section{Fungal material}

The Cortinarius samples (Table 1) were collected in Norway between 1985 and 2008. The sampling is covering the main variation within North European species and is ranging from arctic tundra to broadleaved temperate forests. To give the correct name for clades, the type species for the traditional subgenera and (some) sections are included in the sampling (Clements \& Shear 1931, Singer \& Smith 1946; Moser \& Horak 1975, Høiland 1984, Singer 1986). In addition, some sequences (Table 1) were acquired from the NCBInr nucleotide database (http://www.ncbi.nlm. nih.gov/, as of 12.2012) to broaden our species sample to include some species outside North Europe. Suitable out- 
group species (Table 1) (Agrocybe praecox (Pers.) Fayod, Galerina stordalii A.H. Sm., Hebeloma alpinum (J. Favre) Bruchet, and Phaeogalera stagnina (Fr.) Pegler \& T.W.K. Young) were established based on previous molecular phylogenies of dark spored agarics including Cortinarius (Gulden et al. 2005). The nomenclature follows Index Fungorum (http://www.indexfungorum.org/Names/Names. asp, as of 12.2013). Dried, field-collected specimens are deposited in the fungal herbarium of the Natural History Museum, University of Oslo (O).

\section{DNA extraction and PCR}

DNA was obtained from dried specimens. Various protocols for DNA extraction were used: the 2\% CTAB miniprep method (Murray \& Thompson 1980), the microwave miniprep procedure (Goodwin \& Lee 1993) and the Dynabeads R DNA DirectTM System 1 extraction kit (Rudi et al. 1997). Primer pairs used in the PCR reactions included PNS1/NS41 for the partial nr18S region, ITS5/ITS4 for the complete ITS1-5.8S-ITS2 region, and LR0R/LR5 for the partial nr28S region (White et al. 1990). Template was amplified with PuReTaq Ready-To-Go PCR Beads (GEHealthcare). PCRs were run with the following cycling conditions: $1 \times\left(3 \mathrm{~min} / 94{ }^{\circ} \mathrm{C}\right), 40 \times\left(30 \mathrm{~s} / 94{ }^{\circ} \mathrm{C}, 30 \mathrm{~s} / 50-\right.$ $\left.52{ }^{\circ} \mathrm{C}, 1 \mathrm{~min} / 72^{\circ} \mathrm{C}\right), 1 \times\left(10 \mathrm{~min} / 72^{\circ} \mathrm{C}\right)$.

PCR products were purified using ExoSAP-IT (Amersham Biosciences), prior to sequencing. Sequences were generated with an ABI 3730 high-throughput capillary electrophoresis sequencers using the PCR primers as sequencing primers.

\section{Phylogenetic inference}

The three rDNA genes (18S, 5.8S, and 28S) and the internal transcribed spacer regions (ITS1 and ITS2) were collectively aligned using MAFFTv6 Q-INS-I model (Hofacker et al. 2002, Kiryu et al. 2007, Katoh \& Toh 2008), considering secondary RNA structure (default parameters used) as a criterion for the alignment. The alignment was checked manually using MacClade v4.07 (Maddison \& Maddison 1992), before being inferred with Gblocks v0.91b (Castresana 2000), under the least stringent parameters, to exclude poorly aligned positions and divergent regions from subsequent phylogenetic inferences. The inference of a concatenated rDNA alignment gave a $68 \%$ increase in informative nucleotide characters ( 825 to 491 ) compared to that of inferring just the 5.8S and the ITS regions. Ambiguous species were subsequently excluded from downstream analysis upon evaluation of the alignment and preliminary ML (RAxML) topologies. The dataset was analysed with MODLETEST (Posada \& Crandall 1998) to establish the optimal model of nucleotide evolution; for all alignments the General Time Reversible (GTR) model was preferred for both the Akaike and Bayesian information Criterion (AiC and BiC). Maximum Likelihood (ML) analyses were performed with RAxML-VI-HPCv7.2.6, GTRCAT model with 25 rate categories (Stamatakis 2006). The most likely topology was established from 100 separate searches, and bootstrap analyses were performed with 500 pseudoreplicates. Bayesian analyses were carried out with MrBayes MPI version 3.1.2 (Huelsenbeck et al. 2001, Ronquist \& Huelsenbeck 2003). Trees were generated from two independent runs with one heated and one cold chain in the
Markov Chain Monte Carlo (MCMC) with 40,000,000 generations, sampling every 1000 . Analyses ran until the average standard deviation of split frequencies was $<0.01$. Burn-in trees were set based on the assessment of likelihood plots and convergence diagnostics implemented in MrBayes. The Potential Scale Reduction Factor (PSRF) values for all inferences were $\sim 1.0$, indicating a good posterior probability distribution sample. The majority rule tree and posterior probabilities for each inference was constructed from a consensus of the sampled post burn-in trees. Species with an uncertain phylogenetic affinity (rogue taxa), were established using the RogueNaRok-algorithm (Aberer et al. 2013), with the following parameters; a majority-rule consensus threshold, support optimization and a Max dropset size of 5. Topological congruence between the inferred phylogenies was calculated using the $l_{\text {cong }}$ index: http://max2.ese.u-psud.fr/bases/upresa/pages/devienne/ index.html (de Vienne et al. 2007). All model estimation and phylogenetic analyses, unless otherwise stated, were done on the freely available Bioportal (Kumar et al. 2009) at the University of Oslo (http://www.bioportal.uio.no/, as of 12.2013).

\section{Morphological, chemical, and ecological data}

Morphological and ecological features were acquired from literature (Moser 1983, Moser \& Horak 1975, Ammirati \& Smith 1978, Brandrud et al. 1990-2013, Høiland \& HolstJensen 2000, Niskanen et al. 2008) and our own observations. Information about chemistry was taken from reviews or discrete works dealing with Cortinarius chemistry (Høiland 1984, Gill \& Steglich 1987, Keller et al. 1988, von Nussbaum et al. 1998, Spiteller et al. 2000, Brondz et al. 2007, Brondz \& Høiland 2008, Teichert et al. 2008).

\section{Results}

\section{Sequence amplification and assembly}

\section{DNA sequences from 69 Cortinarius spe-} cies and four outgroup species were successfully amplified for the whole rDNA gene locus, except for $C$. gentilis, which failed for $28 \mathrm{~S}$, and C. torvus, which failed for $18 \mathrm{~S}$. The sequences are deposited in Genbank under the accession

Table 1. DNA sequences used in this study: ${ }^{\text {SSpecies }}$ names follow Index Fungorum (www.indexfungorum.org/ Names/Names.asp, as of 12.2013). ${ }^{\mathrm{b}}$ Accession numbers in EMBL/GenBank/DDBJ (www.ncbi.nlm.nih.gov). m.d. = missing data. Asterisks * indicate sequences generated in this study. The capital letter after the species name author indicates the subgenus/genus in traditional systematics (Moser \& Horak 1975); (Moser 1983). Dermocybe D, Leprocybe L, Myxacium M, Phlegmacium P, Rozites R, Sericeocybe S, and Telamonia T. Hashtags \# denote type species for subgenus/genus. 


\begin{tabular}{|c|c|c|c|c|}
\hline Species $^{a}$ & Geographic origin & $\begin{array}{l}\text { GenBank } \\
\text { acc. no. } \\
18 \text { S region }{ }^{b}\end{array}$ & $\begin{array}{l}\text { GenBank } \\
\text { acc. no. } \\
\text { ITS1-5.8S-ITS2 } \\
\text { region }{ }^{\text {b }}\end{array}$ & $\begin{array}{l}\text { GenBank } \\
\text { acc. no. } \\
28 \text { S region }\end{array}$ \\
\hline Agrocybe praecox (Pers.) Fayod Outgroup & Norway: Hordaland & KC171232* & KC842389* & KC842460* \\
\hline Galerina stordali A.H. Sm. Outgroup & Norway: Hordaland & KC171235* & KC842392* & KC842463* \\
\hline Hebeloma alpinum (J. Favre) Bruchet Outgroup & Norway: Hordaland & KC171234* & KC842391* & KC842462* \\
\hline Phaeogalera stagnina (Fr.) Pegler \& T.W.K. Young Outgroup & Norway: Hordaland & KC171233* & KC842390* & KC842461* \\
\hline Cortinarius acutus (Pers.) Fr. T & Norway: Oslo & KC171262* & KC842420* & KC842490* \\
\hline Cortinarius albonigrellus J. Favre T & Norway: Oppland & KC171239* & KC842396 & KC842467* \\
\hline Cortinarius alboviolaceus (Pers.) Fr. S\# & Norway: Rogaland & KC171244* & KC842402* & KC842473* \\
\hline Cortinarius amoenus (M.M. Moser \& E. Horak) G. Garnier D & Chile: Valdivia & m.d. & AF539721 & AF539721 \\
\hline Cortinarius anomalus (Fr.) Fr. S & Norway: Sogn og Fjordane & KC171267* & KC842425* & KC842495* \\
\hline Cortinarius anserinus (Velen.) Rob. Henry P & Germany: Tondorf & m.d. & AY174805 & AY174805 \\
\hline Cortinarius armeniacus (Schaeff.: Fr.) Fr. T & Norway: Oslo & KC171245* & KC842403* & KC842474* \\
\hline Cortinarius armillatus (Fr.) Fr. T & Norway: Rogaland & KC171250* & KC842408* & KC842479* \\
\hline Cortinarius austroduracinus M.M. Moser T & Chile & m.d. & AY669653 & AY669653 \\
\hline Cortinarius balteatocumatilis Rob. Henry ex P.D. Orton P & France: Piroulette & m.d. & AY174801 & AY174801 \\
\hline Cortinarius balteatus (Fr.) Fr. P & Germany & m.d. & AY669526 & AY669526 \\
\hline Cortinarius bolaris (Pers.) Fr. L & Norway: Telemark & KC171268* & KC842426* & KC842496* \\
\hline Cortinarius brunneus (Pers.) Fr. T & Norway: Oslo & KC171252* & $\mathrm{KC} 842410^{*}$ & KC842480* \\
\hline Cortinarius caerulescens (Schaeff.) Fr. P & Canada: British Columbia & m.d. & HQ604682 & HQ604682 \\
\hline Cortinarius callisteus (Fr.) Fr. L & Norway: Telemark & KC171277* & KC842435* & KC842505* \\
\hline Cortinarius caninus (Fr.) Fr. S & Norway & KC171266 & KC842424* & KC842494* \\
\hline Cortinarius caperatus (Pers.) Fr. R\# & Norway: Oslo & KC171285* & KC842443* & KC842513* \\
\hline Cortinarius cinnabarinus Fr. D & Norway: Vestfold & KC171247* & KC842405* & KC842476* \\
\hline Cortinarius cinnamomeus (L.) Fr. D\# & Norway: Oslo & KC171255* & KC842413* & KC842483* \\
\hline Cortinarius claricolor (Fr.) Fr. P & Norway: Aust-Agder & KC171292* & KC842450* & KC842520* \\
\hline Cortinarius collinitus (Pers.) Fr. M\# & Norway: Nord-Trøndelag & KC171276 & KC842434* & KC842504* \\
\hline Cortinarius colymbadinus Fr. L & Norway: Buskerud & KC171246* & KC842404* & KC842475* \\
\hline Cortinarius cotoneus Fr. L\# & Norway: Oslo & KC171265* & KC842423* & KC842493* \\
\hline Cortinarius crassus Fr. P & Norway: Aust-Agder & KC171279* & KC842437 & KC842507* \\
\hline Cortinarius croceus (Schaeff.) Gray D & Norway: Oslo & KC171256* & KC842414* & KC842484* \\
\hline Cortinarius cumatilis Fr. $\mathrm{P}$ & Norway: Oslo & KC171291* & KC842449* & KC842519* \\
\hline Cortinarius delibutus Fr. M & Norway: Oslo & KC171283* & KC842441* & KC842511* \\
\hline Cortinarius evernius (Fr.) Fr. T & Norway: Oslo & KC171243* & KC842401 ${ }^{*}$ & KC842472* \\
\hline Cortinarius flexipes (Fr.) Fr. T & Norway: Oslo & KC171238* & KC842395* & KC842466* \\
\hline Cortinarius fraudulosus Britzelm. P & Norway: $\varnothing$ stfold & KC171288* & KC842446 & KC842516 ${ }^{*}$ \\
\hline Cortinarius gentilis (Fr.) Fr. L & Norway: Nord-Trøndelag & KC171251* & KC842409* & m.d. \\
\hline Cortinarius glaucopus (Schaeff.) Fr. P & Norway: Oslo & KC171287* & KC $842445^{*}$ & KC $842515^{*}$ \\
\hline Cortinarius icterinus (E. Horak) E. Horak D & Chile: Valdivia & m.d. & AF539720 & AF539720 \\
\hline Cortinarius idahoensis Ammirati \& A.H. Sm. D & Canada: British Columbia & m.d. & FJ039596 & FJ039596 \\
\hline Cortinarius infractus (Pers.) Fr. P & Norway: Oslo & KC171269* & KC842427* & KC842497* \\
\hline Cortinarius laniger Fr. $\mathrm{T}$ & Norway: Telemark & KC171241* & KC842398* & KC842469* \\
\hline Cortinarius limonius (Fr.) Fr. L & Norway: Rogaland & KC171298* & KC842456* & KC842526* \\
\hline $\begin{array}{l}\text { Cortinarius luteostriatulus (M.M. Moser \& E. Horak) E. Valenz. \& G. Moreno D } \\
\text { Cortinarius malicorius Fr. D }\end{array}$ & Chile: Osborno & m.d. & AF539707 & AF539707 \\
\hline $\begin{array}{l}\text { Cortinarius malicorius Fr. D } \\
\text { Cortinarius multiformis (Fr.) Fr. P }\end{array}$ & Austria & & AY669583 & AY669583 \\
\hline Cortinarius napus Fr. P & Norway: Oppland & KC171270* & $\begin{array}{l}\text { KC842458 } \\
\text { KC842428* }\end{array}$ & $\begin{array}{l}\text { KC842528 } \\
\text { KC842498* }\end{array}$ \\
\hline Cortinarius obscurooliveus (M.M. Moser) Kuhn.-Fink. \& Peintner D & Chile: Temuco & m.d. & AF539708 & AF539708 \\
\hline Cortinarius obtusus (Fr.) Fr. T & Norway: Oslo & KC171263* & KC842421* & KC842491* \\
\hline Cortinarius olearioides Rob. Henry P & Norway: Oslo & KC171271* & KC842429* & KC842499* \\
\hline Cortinarius olidus J.E. Lange $P$ & Norway: Oslo & KC171293* & KC842451* & KC842521 ${ }^{*}$ \\
\hline Cortinarius olivaceofuscus Kühner D & Norway: Sogn og Fjordane & KC171259* & KC842417* & KC842487* \\
\hline Cortinarius orellanus Fr. L & Norway: Aust-Agder & KC171261* & KC842419* & KC842489* \\
\hline Cortinarius parvannulatus Kühner T & Norway: Oppland & KC171236* & KC842393* & KC842464* \\
\hline Cortinarius percomis Fr. $\mathrm{P}$ & Norway: Rogaland & KC171294* & KC $842452^{*}$ & KC842522* \\
\hline Cortinarius phaeopygmaeus J. Favre T & Norway: Oppland & KC171240* & KC842397 & KC842468* \\
\hline Cortinarius phoeniceus (Vent.) Maire var. occidentalis A.H. Sm. D & Canada: British Columbia & m.d. & FJ039592 & FJ039592 \\
\hline Cortinarius pholideus (Lilj.) Fr. S & Norway: Oslo & KC171248* & KC842406* & KC842477* \\
\hline Cortinarius piceae Frøslev, T.S. Jeppesen \& Brandrud P & Norway: Oppland & KC171272* & KC842430* & KC842500* \\
\hline Cortinarius polaris Høil. D & Norway: Svalbard & KC171253* & KC $842411^{*}$ & KC842481* \\
\hline Cortinarius porphyropus (Alb. \& Schwein.) Fr. P & Norway: Rogaland & KC171295* & KC $842453^{*}$ & KC842523* \\
\hline Cortinarius pseudoglaucopus (Jul. Schäff. ex M.M. Moser) Quadr. P & Germany & m.d. & AY669573 & AY669573 \\
\hline Cortinarius raphanoides (Pers.) Fr. L & Norway: Buskerud & KC171249* & KC842407 ${ }^{*}$ & KC842478* \\
\hline Cortinarius renidens Fr. T & Norway: Oslo & KC171301* & KC842459* & KC842529* \\
\hline Cortinarius rubellus Cooke L & Norway: Oslo & KC171260* & KC842418* & KC842488* \\
\hline Cortinarius rubicundulus (Rea) A. Pearson L & Norway: Oslo & KC171278* & KC842436* & KC842506 ${ }^{*}$ \\
\hline Cortinarius saginus (Fr.) Fr. P\# & Norway: Telemark & KC171290* & KC842448* & KC842518* \\
\hline Cortinarius salor Fr. M & Norway: Aust-Agder & KC171280* & KC842438* & KC842508* \\
\hline Cortinarius sanguineus (Wulfen) Fr. D & Norway: Oslo & KC171258* & KC842416* & KC842486* \\
\hline Cortinarius scaurus (Fr.) Fr. P & Norway: Rogaland & KC171296* & KC842454* & KC842524* \\
\hline Cortinarius scotoides J. Favre T & Norway: Oppland & KC171237* & KC842394* & KC842465* \\
\hline Cortinarius semisanguineus (Fr.) Gillet D & Norway: Buskerud & KC171257* & KC842415* & KC842485* \\
\hline Cortinarius stillatitius Fr. M & Norway: Oppland & KC171274* & KC842432 ${ }^{*}$ & KC842502* \\
\hline Cortinarius subbalaustinus Rob. Henry T & Sweden & m.d. & AF195592 & AF195592 \\
\hline Cortinarius subtortus (Pers.) Fr. P & Norway: Oslo & KC171281* & KC842439* & KC842509* \\
\hline Cortinarius sulphurinus Quél. P & Norway: Akershus & KC171273* & KC $842431^{*}$ & KC842501* \\
\hline Cortinarius terpsichores Melot P & Norway: Oslo & KC171286* & KC842444* & KC842514* \\
\hline Cortinarius tiliae Brandrud P & Norway: Oslo & m.d. & AY669556 & AY669556 \\
\hline Cortinarius torvus (Fr.) Fr. T\# & Norway: Telemark & m.d. & KC842400* & KC842471* \\
\hline Cortinarius traganus (Fr.) Fr. S & Norway: Oslo & KC171242* & KC842399* & KC842470* \\
\hline Cortinarius triumphans Fr. P & Germany: Ramersbach & m.d. & AY174799 & AY174799 \\
\hline Cortinarius trivialis J.E. Lange M & Norway: Oppland & KC171275* & KC $842433^{*}$ & KC842503* \\
\hline Cortinarius turmalis Fr. P & Norway: Oslo & KC171297* & KC842455* & KC842525* \\
\hline Cortinarius uliginosus Berk. D & Norway: Oslo & KC171254* & KC842412* & KC842482* \\
\hline Cortinarius vacciniophilus Brandrud $\mathrm{P}$ & Norway: Oppland & m.d. & AY669518 & AY669518 \\
\hline Cortinarius variicolor (Pers.) Fr. P & Norway: Akershus & KC171289* & KC842447 ${ }^{*}$ & KC842517* \\
\hline Cortinarius varius (Shaeff.) Fr. P & Germany: Oberjoch & m.d. & AY174792 & AY174792 \\
\hline Cortinarius venetus (Fr.) Fr. L & Norway: Oslo & KC171264* & KC842422 ${ }^{*}$ & KC842492* \\
\hline Cortinarius vespertinus (Fr.) Fr. P & Norway: Aust-Agder & KC171299* & KC $842457^{*}$ & KC842527* \\
\hline Cortinarius vibratilis (Fr.) Fr. M & Norway: Rogaland & KC171282* & KC842440* & KC842510* \\
\hline Cortinarius violaceus (L.) Gray C\# & Norway: Rogaland & KC171284* & KC842442* & KC $842512^{*}$ \\
\hline Cortinarius viridocoeruleus Chevassut $\&$ Rob. Henry P & Germany: Eschweiler & m.d. & AY174788 & AY174788 \\
\hline
\end{tabular}


numbers KC171232-KC171301 and KC842389KC842529 (Table 1). In addition, sequences from 17 species were retrieved from the NCBInr nucleotide database (Table 1).

Alignments generated in this study have been made freely available through the authors ResearchGate pages (http://www.researchgate.net/).

\section{Phylogenetic inference}

The following species were pruned upon evaluation of the RogueNaRok-algorithm (Aberer et al. 2013): Cortinarius rubicundulus (Rea) A. Pearson, $C$. saginus, $C$. salor Fr., and C. callisteus (Fr.) Fr. However, pruning gave no additional support (data not shown). The inferred Cortinarius phylogenies (pruned or non-pruned analysed with ML or MrBayes, respectively) demonstrated good topological congruence with an $l_{\text {cong }} \mathrm{P}$-value $<0.05$. Removal of long-branching species, likewise, had minimal topological impact (data not shown). For this reasoning, the full dataset was preferred for visualization of the results. For interpretation of the phylogenetic inferences (Fig. 1), statistical support is defined as: full 1.00 posterior probability (PP)/100 bootstrap support (BS), high $>90 \mathrm{BS}$, moderate $>65 \mathrm{BS}$, and low $>50 \mathrm{BS}$, highlighted with red lines in Fig. 1. Additionally, PP support over 0.95 in cases where $\mathrm{BS} \leq 50$ is highlighted with blue lines in Fig. 1.

The first and most basal clade to diverge from the main branch constituted $C$. austroduracinus M.M. Moser and C. renidens Fr. However, the basal position and the monophyly of these species were unsupported. The next grouping to diverge, again unsupported, was a lineage encompassing the terminal Scauri clade, recovered with moderate support (1.00/66). The following divergence was the Calochroi clade: The split was unsupported, however the grouping of the Calochroi clade was highly supported (1.00/96), as was the sister relationship between $C$. olearioides Rob. Henry and $C$. napus Fr. (1.00/94). The subsequent unsupported divergence was a large grouping harbouring the Phlegmacium and Myxacium lineages. The branching pattern between species was uncertain with lack of support for relationships apart from; the fully supported monophyly of the Myxacium clade, the low supported clade formed by C. glaucopus (Schaeff.) Fr., C. caerulescens (Schaeff.) Fr.,
C. terpsichores Melot, C. anserinus (Velen.) Rob. Henry, and $C$. viridocoeruleus Chevassut \& Rob. Henry $(0.98 / 52)$, and the moderate to highly supported sister relationships recovered between $C$. cumatilis Fr. and C. claricolor (Fr.) Fr. (1.00/96), C. olidus J.E. Lange and C. percomis Fr. (1.00/88), and $C$. balteatocumatilis Rob. Henry ex P.D. Orton and C. balteatus (Fr.) Fr. (1.00/98). The next species to split from the main branch was $C$. callisteus, subsequently followed by $C$. infractus, with both lacking support. The ensuing branch to split formed the unsupported Anomali clade. Only the

Figure 1. The rDNA phylogeny of Cortinarius.

ML tree for 86 Cortinarius species and four outgroup species (2373 rDNA characters). Red lines full $(=100)$ to low BS $(>50)$; blue lines $\mathrm{PP} \geq 0.95$ for $\mathrm{BS} \leq 50$. Main clades are named after subgenera or sections, sub-clades are named with letters.

Letter(s) following a species names indicates type species for genus Rozites R, subgenera Dermocybe D, Leprocybe L, Myxacium M, Phlegmacium P, Sericeocybe S, and Telamonia T, and sections Icterinula I, Olivaceofusci O, and Pauperae $\mathrm{Pa}$.

Morphological, chemical, and ecological traits (blank = actual characteristic is lacking). Viscid: $\mathrm{P}=$ pileus, $\mathrm{PS}=$ pileus and stipe; spore shape: $\mathrm{E}=$ ellipsoid- amygdaloid, $\mathrm{S}=$ subglobose, $\mathrm{C}=$ citriniform; cuticle: $\mathrm{S}=$ simple, $\mathrm{D}=$ duplex, $\mathrm{DA}=$ duplex and amyloid, $?=$ no data; octaketide dimeric pre-anthraquinones: $\mathrm{F}=$ flavomannins, $\mathrm{P}=$ phlegmacins, $\mathrm{H}=$ hypericin and skyrin; octaketide monomeric anthraquinones: $\mathrm{E}=$ only endocrocin, $\mathrm{EO}$ $=$ endocrocin and other anthraquinones, $\mathrm{O}=$ only other anthraquinones; nitrogen compounds: $\mathrm{O}=$ orellanine, $\mathrm{C}$ $=1: 2$ iron (III) (R)- $\beta$-dopa complex, $\mathrm{I}=$ infractopicrin, $\mathrm{Q}=$ (iso)-quinoline alkaloids; mycorrhizal partner(s): $\mathrm{B}=$ Betula, $\mathrm{C}=$ conifers, $\mathrm{CB}=$ conifers and Betula, $\mathrm{CBD}=$ conifers and broad leaved deciduous trees, $\mathrm{D}=$ deciduous trees, $\mathrm{BD}=$ broad leaved deciduous trees, $\mathrm{I}=$ indifferent, $\mathrm{P}=$ Picea, $\mathrm{N}=$ Nothofagus, $\mathrm{S}=$ Salix; soil: $\mathrm{P}=$ poor, $\mathrm{M}=$ medium, $\mathrm{R}=$ rich, $\mathrm{C}=$ calcareous, $?=$ no data; special characteristics: $1=$ balloon shaped cells on lamella, 2 = flesh yellowing, $3=$ cystidia with epiparietal pigment, 4 = ring, ectomycorrhizae with amyloid mantle, $5=$ cystidia with bluish pigment, spores with plage, $6=$ flesh yellowing, clavate cheilocystidia, spores indistinctly verrucose, $7=$ cylindrical cystidia, $8=$ flesh purple in $\mathrm{KI}_{3}$, $9=$ crystallopicrin, $10=$ basal mycelium staining rose, spores smooth, $11=$ veil and cortina absent. 


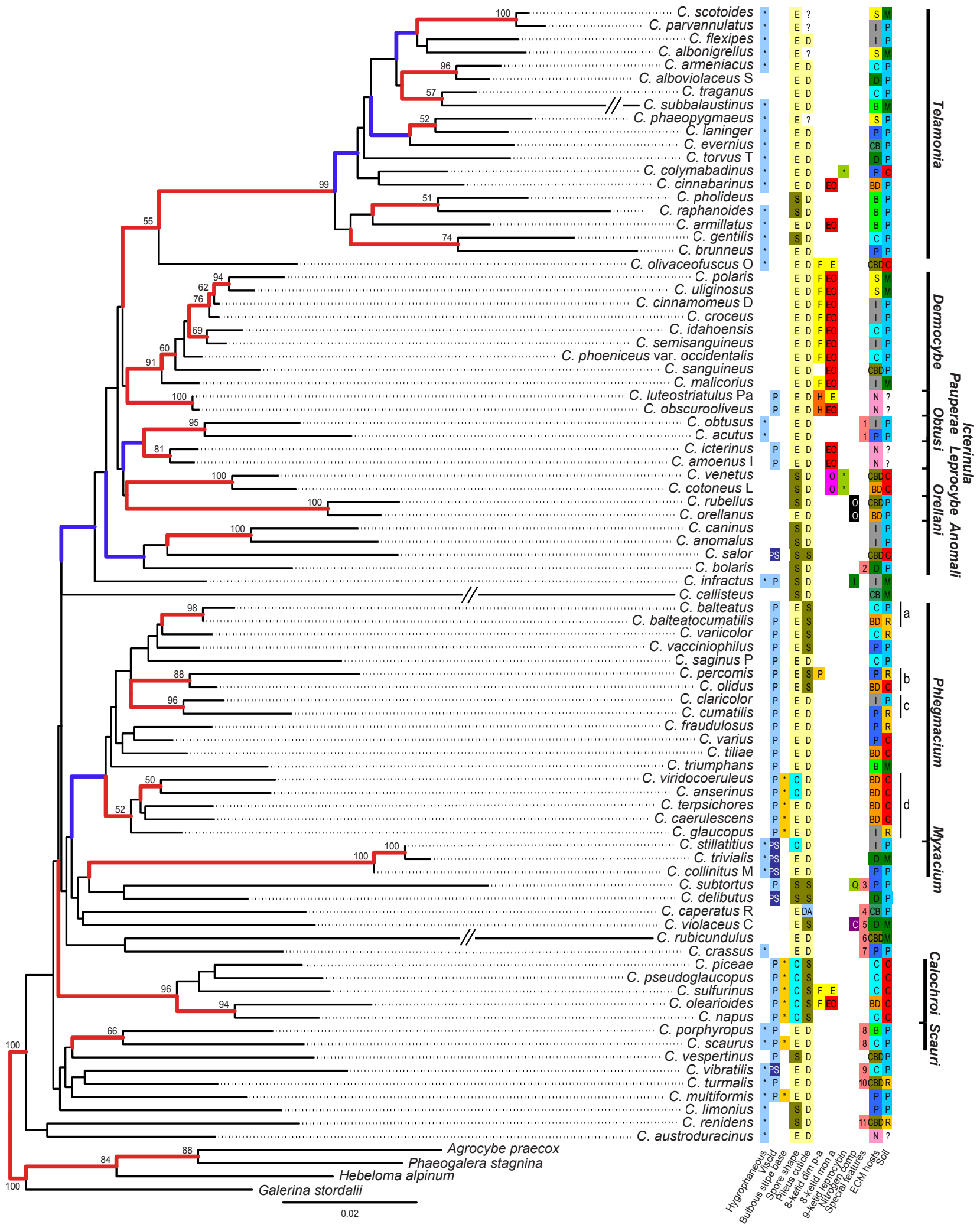


sister relationship of $C$. anomalus (Fr.) Fr. and $C$. caninus (Fr.) Fr. was confirmed with full support. The next grouping to diverge, again with lacking support, constituted four supported sister relationships; the fully supported Orellani clade, the fully supported Leprocybe clade, the moderately supported Icterinula clade (1.00/81), and the highly supported Obtusi clade (1.00/95). The subsequent unsupported divergence constituted a grouping where the fully supported Pauperae clade split first, recovering the highly supported Dermocybe clade (1.00/91). The ensuing divergence of C. olivaceofuscus Kühner from the main branch was inferred with low support (0.99/55). The subsequent split was highly supported (1.00/99), constituting the Telamonia clade. The branching pattern in the clade, however, was unclear with few sister relationships being recovered with support; $C$. gentilis with C. brunneus (1.00/74), C. pholideus (Lilj.) Fr. with C. raphanoides (Pers.) Fr. (0.99/51), C. phaeopygmaeus J. Favre with C. laniger Fr. (0.99/52), C. traganus (Fr.) Fr. with C. subbalaustinus Rob. Henry (0.99/57), C. armeniacus with C. alboviolaceus (1.00/96), and finally the fully supported $C$. scotoides J. Favre with $C$. parvannulatus Kühner.

\section{Discussion}

\section{Phylogeny}

Although the branching pattern between clades might be questionable due to uncertain phylogenetic signal, twelve infrageneric groups received satisfactory support (Fig. 1). These are: Telamonia, Dermocybe, Pauperae, Obtusi, Icterininula, Leprocybe, Orellani, Anomali, Phlegmacium, Myxacium, Calochroi, and Scauri. As mentioned in the introduction chapter most of these groups have recurrently been identified by several authors, e.g. (Høiland \& Holst-Jensen 2000; Garnica et al. 2003a, 2003b, 2005; Peintner et al. 2004; Niskanen 2008; Harrower et al. 2011). Our results confirm their findings, and except for Anomali and Phlegmacium, the phylogeny will not be discussed further.

Our analyses show that $C$. olivaceofuscus is recovered, with low BS, as a sister group to Telamonia, and we may discuss whether it should be included in Telamonia or not. Previously it has been included in Dermocybe section Olivaceofusci
(Høiland 1984). With Telamonia it shares a hygrophaneous pileus, with Dermocybe the octaketide anthraquinones typical for this group (Høiland 1984, Gill \& Steglich 1987). Based on morphology and pigment chemistry C. olivaceofuscus has only one known relative, Dermocybe leptospermarum E. Horak (not yet combined in Cortinarius) from New Zealand (Høiland 1984, Gill \& Steglich 1987, Horak 1988, Keller et al. 1988). It is tempting to consider this small, strictly bi-Hemispheric group as an evolutionary link between Dermocybe and Telamonia, representing the ancestral state of the latter. The sequence of $C$. olivaceofuscus included in the Dermocybe clade by Garnica et al. (2005) is not matching this species, but is similar to $C$. cinnamomeus (BLASTn hit with $99 \%$ identity and/or coverage, as of 09.2014).

The Bayesian inference supports a relationship between clade Obtusi and Icterinula (0.95/46), but no recognised morphological or chemical features combine these two clades. The same relationship was also shown by a previous analysis (Peintner et al. 2004).

Clade Anomali in its most narrow sense comprises $C$. anomalus and C. caninus, characterised by subglobose spores and dry basidiocarps often with glimmery pileus. Anomali was originally recognised as a section of subgenus Telamonia or alternatively subgenus Sericeocybe (Moser 1983, Niskanen et al. 2008). The European C. spilomeus (Fr.) Fr. and the South Hemispheric C. tristis E. Horak and C. sclerophyllarum Gasparini are added to this lineage (Garnica et al. 2005). According to the Bayesian tree, the clade can be broadened to include $C$. salor and C. bolaris (Pers.) Fr. (0.99/32). Cortinarius salor shows morphological similarity to $C$. delibutus Fr. by its slimy stipe and pileus, with which some earlier phylogenetic analyses indicate a possible relationship (Peintner et al. 2004, Harrower et al. 2011, Ryberg \& Matheny 2011). Our result does not support this relationship. The inclusion of $C$. bolaris in clade Anomali seems, however, more justified based on morphological features (Garnica et al. 2005, Niskanen et al. 2008). A chemically unknown compound staining the flesh yellow, is characteristic for this species (Høiland 1980).

Cortinarius infractus is tentatively placed as a sister group to the assemblage spanning from clade Telamonia to Anomali. It has a Phlegmacium-like basidiocarp with a viscid pileus, subglo- 
bose spores, and contains indole alkaloids (Brondz et al. 2007, Brondz \& Høiland 2008), probably unique for Cortinarius.

It is worth noting that the Bayesian phylogeny gives support to a "superclade" $(0.98 / 16)$ spanning from clade Telamonia to clade Anomali, including $C$. infractus. In this superclade we find the majority of species with soluble pigments of octaketide origin, all species with compounds of nonaketide origin, the majority of species with hygrophaneous pileus, few species with viscid pileus, and no species with bulbous stipe base. Moreover, all species (except $C$. salor) have duplex pileus cuticle.

Interestingly, it is demonstrated that the ectomycorrhizal structure of species in clade Dermocybe, Obtusi, and Leprocybe possesses emanating hyphae closed by a clamp, a feature not seen in the other clades (although only a limited number of species were investigated) (Agerer 2006). This may confirm the relationship between these clades. Species from the other clades have emanating hyphae with open anastomoses, except for C. caperatus (Pers.) Fr. in which they are smooth with clamps (Agerer 2006).

Clade Phlegmacium is not supported by ML, however, the Bayesian inference provides support $(0.97 / 28)$ for this large group spanning from the couple C. balteatus and C. balteatocumatilis to C. glaucopus. This clade may represent the retained part of subgenus Phlegmacium since Cortinarius saginus, the type species (Singer 1986), is a member of the group. Common for all these species is a viscid pileus, a dry stipe, and stout basidiocarps. Inside this clade ML gives support to some subordinate monophyletic groups marked a, b, c, and d in Fig. 1 .

Cortinarius balteatus and C. balteatocumatilis (sub-clade a) are sister species with high BS support. Although not supported by ML or Mr. Bayes, both analyses are congruent in inferring a monophyletic relationship with C. variicolor (Pers.) Fr. and $C$. vacciniophilus Brandrud. This broadened concept of sub-clade a represents clade Phlegmacioides (Garnica et al. 2005).

Cortinarius percomis and C. olidus are monophyletic with moderate BS support (sub-clade b). This clade also includes species as $C$. nanceiensis Maire, C. mussivus (Fr.) Melot, and C. papulosus Fr. represening Percomes (Garnica et al. 2005). Cortinarius percomis, $C$. nanceiensis, and $C$. mussivus contain special octaketide pigments not found outside this group (Gill \& Steglich 1987).
Cortinarius claricolor and C. cumatilis (subclade c) are sister species with high BS support, representing Praestantes (Garnica et al. 2005).

The group spanning from the couple $C$. viridocoeruleus and $C$. anserinus to $C$. glaucopus forms a monophyletic clade with low BS support (sub-clade d). A monophyletic relationship between these species in this, still unnamed, clade has also previously been demonstrated (Garnica et al. 2003b).

A number of species in our dataset are found in unsupported groups. Cortinarius violaceus contains a violet iron complex, unique for this species (von Nussbaum et al. 1998, Spiteller et al. 2000). Cortinarius caperatus has, in addition to the membranous ring, an amyloid reaction in the cutis hyphae and the ectomycorrhizal mantle together with smooth emanating hyphae with clamps (Agerer 2006). Despite forming an unsupported monophyly, the very dissimilar morphological and chemical characteristics indicate that $C$. violaceus and $C$. caperatus are not closely related, thus this can be considered a topological artefact. The couple C. subtortus and C. delibutus constitute another unconfirmed monophyletic group. Common for both species is a slimy pileus and subglobose spores. Cortinarius subtortus has in addition prominent pleuro- and cheilocystidia and content of (iso)-quinoline alkaloids (Teichert et al. 2008). Cortinarius delibutus has additionally a slimy stipe. Cortinarius rubicundulus and $C$. crassus Fr. is by ML, but not by Mr. Bayes, put in an unsupported group. They share some morphological similarities such as stout basidiocarps and cystidia, but the spore ornamentation is different; indistinctly verrucose in C. rubicundulus, distinctly verrucose in $C$. crassus. Moreover $C$. rubicundulus contains a chemically unknown compound staining the flesh yellow, reminiscent to C. bolaris (Høiland 1980). Cortinarius callisteus is by ML put in an isolated, single position and by Mr. Bayes in an unsupported group together with $C$. rubicundulus. Unique for C. callisteus (and its close relative C. citrinofulvescens M.M. Moser) is a strong and peculiar smell of ozone (Niskanen et al. 2008). Both C. callisteus and C. rubicundulus have exceptionally long evolutionary branch lengths, as also shown by other authors (Peintner et al. 2004, Garnica et al. 2005, Harrower et al. 2011, Ryberg \& Matheny 2011).

The species in clade Scauri, together with $C$. 
vespertinus (Fr.) Fr., C. vibratilis (Fr.) Fr., C. turmalis Fr., C. multiformis (Fr.) Fr., and C. limonius (Fr.) Fr., occupy basal positions in the phylogenetic tree. Few characteristics combine these species, but many of them have unique features not seen in the other species of the dataset (Fig. 1). For instance, the viscid pileus cuticle of $C$. vibratilis contains the intensely acrid triterpenoid crystallopicrin (Steglich et al. 1990). The most basal position is taken by the unsupported couple $C$. renidens (characterised by lacking veil and cortina) and the South American Nothofagus associated species $C$. austroduracinus. This monophyletic relationship is also indicated earlier (Garnica et al. 2005).

\section{Morphological characteristics}

A hygrophaneous pileus is dominating among the species in clade Telamonia together with its sister C. olivaceofuscus. It is also met with in clades Obtusi and Myxacium, although weak in the last clade. It is worth noting that hygrophanity is also regular among the more basal species spanning from the couple $C$. austroduracinus and $C$. renidens to clade Scauri. Therefore we consider that hygrophanity is an ancestral character state in genus Cortinarius, which has been retained in some phylogenetic groups and reduced in other groups. The clades Dermocybe, Phlegmacium, and Calochroi are the most prominent examples of non-hygrophaneous groups.

Conversely, a slimy pileus or a slimy pileus and stipe seem derived. This morphological feature seems to have developed independently on multiple occasions in our phylogeny and represents a key characteristic for clades Pauperae, Icterinula, Phlegmacium, Calochroi, and Scauri. The combination slimy pileus and stipe has developed four times in our phylogeny and is a key characteristic for clade Myxacium. It is tempting to believe that the slimy layers have developed to protect the basidiocarps against fungivorous insects and/or temporarily dry weather conditions.

A bulbous stipe base is also considered derived and has probably been evolved due to a pileocarp (subterranean) development as an adaptation to dry soils. It is found in all species in clade Calochroi and sub-clade d of clade Phlegmacium. In our phylogeny this characteristic has evolved on four separate occasions.
The ellipsoid to amygdaloid spores may represent the ancestral spore outline in genus Cortinarius since this outline is dominating in the lower branches of the tree (Fig. 1). Subglobose spores have evolved several times and do not seem to infer a good phylogenetic signal, except in clade Leprocybe and clade Anomali (in its widest sense). Citriniform spores are distinctive for clade Calochroi, but are also encountered in clade Phlegmacium (subclade d) and clade Myxacium. It could be speculated whether the relatively voluminous citriniform spores represent an adaptation to dry environments (Kauserud et al. 2011, Høiland 2012).

A duplex pileus cuticle may also represent an ancestral character state from which a simple cutis has evolved six independent times in our phylogeny. The simple cutis is distinctive for clade Calochroi and also for sub-clades a and b of clade Phlegmacium. Cortinarius caperatus is outstanding by its amyloid duplex cutis (Agerer 2006).

\section{Secondary chemistry}

The most conspicuous secondary compounds in genus Cortinarius are the anthraquinones of the octaketide biosynthetic pathway (Gill \& Steglich 1987). They are distinctive for clades Dermocybe, Pauperae, Icterinula, and Leprocybe, but are also encountered in C. olivaceofuscus, and sporadically in clade Telamonia, Phlegmacium (sub-clade b), and Calochroi. Typical for clade Dermocybe (except C. sanguineus (Wulfen) Fr.), C. olivaceofuscus, and some species in clade Calochroi is content of dimeric pre-anthraquinones of flavomannin type. The dimeric pre-anthraquinones hypericin and skyrin are regularly found in clade Pauperae. In sub-clade b of clade Phlegmacium, dimeric pre-anthraquinones of phlegmacin type are found in $C$. percomis in our phylogeny, but they are also found in the related C. nanceiensis and C. mussivus (Gill \& Steglich 1987, Garnica et al. 2005). Various monomeric anthraquinones, such as endocrocin, dermolutein, and dermorubin, are found in all species of clade Dermocybe, Pauperae, Icterinula, and Leprocybe, and in C. olivaceofuscus (only endocrocin), and the two species of clade Calochroi that also contain flavomannin pre-anthraquinones. The momomeric anthraquinones emodin, dermoglaucin, and dermocybin are only found in clade Dermocybe. In clade Icterinu- 
$l a$ and Leprocybe, together with $C$. cinnabarinus Fr. and C. armillatus (Fr.) Fr. in clade Telamonia, only monomeric anthraquinones are found, no dimeric pre-anthraquinones.

The anthraquinones may act as deterrents to insects and other animals feeding on basidiocarps since it has been demonstrated that anthraquinones in plants (many of them similar to those in fungi, e.g. emodin) have defensive effects upon insects or birds (Trial \& Dimond 1979, Michael et al. 1997). Focusing on clade Dermocybe it is therefore interesting that the basal lines, spanning from $C$. malicorius to the couple $C$. semisanguineus (Fr.) Gillet and C. idahoensis Ammirati \& A.H. Sm., have a broad range of anthraquinones, including emodin, dermoglaucin, and dermocybin. The aforementioned species are found in lowland boreal forests (Høiland 1984) rich in fungivorous insects, and they are lacking in arctic-alpine environments. Presumably, these anthraquinones may have been reduced in the derived lineage leading to the group spanning from $C$. croceus (Schaeff.) Gray to the couple C. uliginosus Berk. and C.polaris Høil. This group only contains endocrocin, dermolutein, and dermorubin, which are universal for all members of Dermocybe, and penetrate the cooler, boreal to arctic-alpine environments with less fungivorous insects. For instance, the fungivorous Mycetophilidae, fungus gnats, are particularly species rich in lowland boreal North European forests, but with only few species in alpine environments (Søli \& Kjærandsen 2008).

Compounds of nonaketide biosynthetic origin, i.e. the strongly fluorescent xanthones leprocybin and leprocyboside, and the monomeric anthraquinone leprolutein, are distinctive for clade Leprocybe (Gill \& Steglich 1987). However, these compounds (except leprolutein) are also found in one species, C. colymbadinus, in clade Telamonia. Like anthraquinones, the xanthones may act as feeding deterrents against insects, as demonstrated for plants (Larson et al. 2010).

An interesting question is whether the possession of the recorded octaketide and nonaketide compounds represents a derived or ancestral character state. Since the actual substances are not seen in the most basal lines in our phylogeny, we may hypothesise that the octaketide pigments were evolved after the split leading to species span- ning from clade Telamonia to clade Calochroi, although the phylogenetic support for this split is limited. The nonaketide compounds may have been evolved later, perhaps after the split leading to species spanning from clade Telamonia to clade Orellani. Later, the biosyntheses of octaketides or nonaketides may have switched off in many evolutionary lineages, and sometimes replaced by biosyntheses of other compounds, such as substances with nitrogen. A hypothesis has been put forward that the possession of pigments in large quantities in species of Calochroi is an ancestral state for this clade, giving rise to species with less or no pigments (Frøslev et al. 2007).

Clade Telamonia is, with few exceptions, remarkably "empty" concerning pigments with octaketide or nonaketide pathways. However, our phylogeny indicates that it probably has originated from fungi possessing such pathways, shown by its sister species, C. olivaceofuscus, and the few Telamonia species containing octaketide or nonaketide substances.

Secondary metabolites containing nitrogen are represented in clade Orellani, with the bipyridines orellanine and orelline (Schumacher \& Høiland 1983, Gill \& Steglich 1987), in the single lines leading to $C$. infractus, with the indole alkaloids pre-infractin ( $\beta$-carboline-1-propionic acid) and infractopicrin (Brondz et al. 2007, Brondz \& Høiland 2008), in C. subtortus with (iso)-quinoline alkaloids (Teichert et al. 2008), and in C. violaceus with an 1:2 iron (III) (R)- $\beta$-dopa complex (von Nussbaum et al. 1998, Spiteller et al. 2000). The metabolites infer good phylogenetic signals for the clades and species at hand, but their diverse chemical nature and erratic occurrence indicate that they have evolved several independent times via different biosynthetic pathways; probably as a response to environmental conditions. The bitter taste due to the alkaloids in $C$. infractus and $C$. subtortus may protect the basidiocarps from being eaten, since animals usually avoid this (Steglich et al. 1990, Spiteller 2008). The same applies to the non-nitrogen compound crystallopicrin in $C$. vibratilis. Orellanine in C. rubellus and C. orellanus may have evolved as a response of enhanced soil acidity and liberation of aluminum ions in soil water since it is a good aluminum ion chelator (Høiland 1994). 


\section{Ecology}

The majority of species in clade Telamonia and Dermocybe prefer poor, acidic coniferous or Betula forests or they are indifferent of forest type. The same applies to the few investigated species of clade Obtusi, Anomali, Myxacium, and Scauri. Several species of Telamonia and a few of Dermocybe, Obtusi, Anomali, and Myxacium have emerged from boreal forests into arctic-alpine environments where they established mycorrhiza with Salix, Betula nana, Dryas, or Bistorta vivipara (Høiland 1984, Gulden \& Torkelsen 1996, Peintner 2008, Bjorbækmo et al. 2010, Geml et al. 2012). Clade Telamonia is remarkably species rich in boreal coniferous forests (Brandrud et al. 1990-2013; Kytövuori et al. 2005; Lindström et al. 2008; Niskanen 2008; Niskanen et al. 2008, $2009,2011,2013 a)$, and we expect these forests to represent vigorous evolutionary arenas for clade Telamonia.

Contrary, clade Leprocybe and Calochroi, and sub-clade $\mathrm{b}$ and $\mathrm{d}$ of Phlegmacium prefer richer forest types, often on calcareous soils (Brandrud et al. 1990-2013, Niskanen et al. 2008). The many species in clade Calochroi are exclusively Northern Hemispheric (Garnica et al. 2009), usually preferring calcareous, dry soils in well-established low boreal coniferous or temperate broadleaved (Fagaceae) forests (Frøslev et al. 2007). These forest types may represent vigorous evolutionary arenas for clade Calochroi. Species in clade Calochroi and sub-clade d of Phlegmacium are rare and have narrow ecological preferences, and many are included in national red lists in Europe (Brandrud et al. 1990-2013, Frøslev et al. 2007). Calochroi may have relatively ancient evolutionary history with a tentative stem about 30 million years (Ryberg \& Matheny 2011).

Clade Pauperae and clade Icterinula are exclusively Southern Hemispheric with preference for mycorrhiza with Nothofagus (Moser \& Horak 1975, Keller et al. 1988).

The two species in clade Orellani have different ecology. Cortinarius orellanus is found in warm and dry Mediterranean to temperate broadleaved (Fagaceae) forests, while C. rubellus prefers cool and moist boreal coniferous forests, seldom under Fagaceae (Høiland 1980, Brandrud et al. 1990-2013, Niskanen et al. 2008). However, a common trait is that both are specialised to acidic soils (Brandrud et al. 1990-2013, Høiland 1994). Very few orellanine containing Cortinarius species are known on global scale, but they occur in both hemispheres (Moser \& Horak 1975, Gasparini 2004). The split between $C$. orellanus and $C$. rubellus is set about 10 million years and the tentative stem for the Orellani group about 20 million years (Ryberg \& Matheny 2011). Orellani may represent an isolated evolutionary lineage of which only a few species have survived up to now (Gasparini 2004).

Acknowledgements: This study was financially supported by the NFR grant 164731 /V40 to TS and ØS. We also acknowledge the ABI lab, Department of Biosciences, University of Oslo, for sequencing service. We are obliged to Cecilie Mathiesen for considerable laboratory help during this project.

\section{References}

Aberer, A.J., Krompass, D. \& Stamatakis, A. 2013: Pruning Rogue Taxa Improves Phylogenetic Accuracy: An Efficient Algorithm and Webservice. - Systematic Biology 62: $162-166$.

Agerer, R. 2006: Fungal relationships and structural identity of their ectomycorrhizae. - Mycological Progress 5: 67-107.

Ammirati, J.F. \& Smith, A.H. 1978: Studies in the genus Cortinarius, IV: Section Dermocybe, new North American species. - Mycotaxon 7: 256-264.

Bjorbækmo, M., Carlsen, T., Brysting, A., Vrålstad, T., Høiland, K., Ugland, K., Geml, J., Schumacher, T. \& Kauserud, H. 2010: High diversity of root associated fungi in both alpine and arctic Dryas octopetala. - BMC Plant Biology 10: 244. doi:210.1186/1471-2229-11101244.

Brandrud, T.E., Lindström, H., Marklund, H., Melot, J. \& Muskos, S. 1990-2013: Cortinarius Flora Photographica. Härnösand.

Brondz, I., Ekeberg, D., Høiland, K., Bell, D.S. \& Annino, A.R. 2007: The real nature of the indole alkaloids in Cortinarius infractus: Evaluation of artifact formation through solvent extraction method development. - Journal of Chromatography A 1148: 1-7.

Brondz, I. \& Høiland, K. 2008: Chemotaxonomic differentiation between Cortinarius infractus and Cortinarius subtortus by supercritical fluid chromatography connected to a multi-detection system. - Trends in Chromatography 4: 79-87.

Castresana, J. 2000: Selection of conserved blocks from multiple alignments for their use in phylogenetic analysis. - Molecular Biology and Evolution 17: 540-552.

Chambers, S.M., Sawyer, N.A. \& Cairney, J.W.G. 1999: Molecular identification of co-occurring Cortinarius and Dermocybe from southeastern Australian sclerophyll forests. - Mycorrhiza 9: 85-90. 
Clements, F.E. \& Shear, C.L. 1931: The genera of fungi. Hafner Publishing Co. New York.

Danel, V.C., Saviuc, P.F. \& Garon, D. 2001: Main features of Cortinarius spp. Poisoning: a literature review. - Toxicon 39: 1053-1060.

Danks, M., Lebel, T. \& Vernes, K. 2010: 'Cort short on a mountaintop' - Eight new species of sequestrate Cortinarius from sub-alpine Australia and affinities to sections within the genus. - Persoonia 24: 106-126.

de Vienne, D.M., Giraud, T. \& Martin, O.C. 2007: A congruence index for testing topological similarity between trees. - Bioinformatics 23: 3119-3124.

Frøslev, T.G., Jeppesen, T.S., Læssøe, T. \& Kjøller, R. 2007: Molecular phylogenetics and delimitation of species in Cortinarius section Calochroi (Basidiomycota, Agaricales) in Europe. - Molecular Biology and Evolution 44: 217-227.

Frøslev, T.G., Matheny, P.B. \& Hibbett, D.S. 2005: Lower level relationships in the mushroom genus Cortinarius (Basidiomycota, Agaricales): A comparison of RPB1, RPB2, and ITS phylogenies. - Molecular Biology and Evolution 37: 602-618.

Garnica, S., Weiss, M. \& Oberwinkler, F. 2003a: Morphological and molecular phylogenetic studies in South American Cortinarius species. - Mycological Research 107: 1143-1156.

Garnica, S., Weiss, M., Oertel, B. \& Oberwinkler, F. 2003b: Phylogenetic relationships of European Phlegmacium species (Cortinarius, Agaricales). - Mycologia 95: $1155-1170$.

Garnica, S., Weiss, M., Oertel, B., Ammirati, J. \& Oberwinkler, F. 2009: Phylogenetic relationships in Cortinarius, section Calochroi, inferred from nuclear DNA sequences. - BMC Evolutionary Biology 9: 1-17.

Garnica, S., Weiss, M., Oertel, B. \& Oberwinkler, F. 2005: A framework for a phylogenetic classification in the genus Cortinarius (Basidiomycota, Agaricales) derived from morphological and molecular data. - Canadian Journal of Botany 83: 1457-1477.

Gasparini, B. 2004: Cortinarius subgenus Orellani in Australia and in the world. - The Australasian Mycologist 23: 62-76.

Geml, J., Timling, I., Robinson, C.H., Lennon, N., Nusbaum, H.C., Brochmann, C., Noordeloos, M.E. \& Taylor, D.L. 2012: An arctic community of symbiotic fungi assembled by long-distance dispersers: phylogenetic diversity of ectomycorrhizal basidiomycetes in Svalbard based on soil and sporocarp DNA. - Journal of Biogeography 39: 74-88.

Gill, M. \& Steglich, W. 1987: Pigments of Fungi (Macromycetes). - Progress in the Chemistry of Organic Natural Products 51: 1-137.

Goodwin, D.C. \& Lee, S.B.B. 1993: Microwave miniprep of total genomic DNA from fungi, plants, protists and animals for PCR. - BioTechniques 15: 438, 441-442, 444.

Gulden, G., Stensrud, Ø., Shalchian-Tabrizi, K. \& Kauserud, H. 2005: Galerina Earle: A polyphyletic genus in the consortium of dark-spored agarics. - Mycologia 97: 823-837.

Gulden, G. \& Torkelsen, A.-E. 1996: Part 3. Fungi I. Basidiomycota: Agaricales, Gasteromycetales, Aphyllophorales, Exobasidiales, Dacrymycetales and Tremellales.
- In: Elvebakk, A. \& Prestrud, P. (eds). A catalogue of Svalbard plants, fungi, algae and cyanobacteria, 173206. Norsk Polarinstitutt Skrifter. Oslo.

Harrower, E., Ammirati, J.F., Cappuccino, A.A., Ceska, O., Kranabetter, J.M., Kroeger, P., Lim, S., Taylor, T. \& Berbee, M.L. 2011: Cortinarius species diversity in British Columbia and molecular phylogenetic comparison with European specimen sequences. - Botany 89: 799-810.

Hofacker, I.L., Fekete, M. \& Stadler, P.F. 2002: Secondary structure prediction for aligned RNA sequences. - Journal of Molecular Biology 319: 1059-1066.

Horak, E. 1988: New species of Dermocybe (Agaricales) from New Zealand. - Sydowia 40: 81-122.

Huelsenbeck, J.P., Ronquist, F., Nielsen, R. \& Bollback, J.P. 2001: Bayesian inference of phylogeny and its impact on evolutionary biology. - Science 294: 2310-2314.

Høiland, K. 1980: Cortinarius subgenus Leprocybe in Norway. - Norwegian Journal of Botany 27: 101-126.

Høiland, K. 1984: Cortinarius subgenus Dermocybe. - Opera Botanica 71: 1-113.

Høiland, K. 1994: Suppression of the toxic effect of soluble aluminium on fungi by dermocybin- $1-\beta-\mathrm{D}$ glucopyranoside and orellanine from Cortinarius sanguineus and C. orellanoides. - Nordic Journal of Botany 14: 221-228.

Høiland, K. 2012: An investigation of basidiospore characteristics in sand dune mushrooms from Lista, Southwestern Norway. - Agarica 32: 49-58.

Høiland, K. \& Holst-Jensen, A. 2000: Cortinarius phylogeny and possible taxonomic implications of ITS rDNA sequences. - Mycologia 92: 694-710.

Katoh, K. \& Toh, H. 2008: Improved accuracy of multiple ncRNA alignment by incorporating structural information into a MAFFT-based framework. - Bioinformatics 9: 212.

Kauserud, H., Heegaard, E., Halvorsen, R., Boddy, L., Høiland, K. \& Stenseth, N.C. 2011: Mushroom's spore size and time of fruiting are strongly related: is moisture important? . - Biology Letters 7: 273-276.

Keller, G., Moser, M., Horak, E. \& Steglich, W. 1988: Chemotaxonomic investigations of species of Dermocybe (Fr.) Wünsche (Agaricales) from New Zealand, Papua New Guinea and Argentina. - Sydowia 40: 168-187.

Kiryu, H., Kin, T. \& Asai, K. 2007: Robust prediction of consensus secondary structures using averaged base pairing probability matrices. - Bioinformatics 23: 434441.

Kumar, S., Skjaeveland, A., Orr, R.J.S., Enger, P., Ruden, T., Mevik, B.-H., Burki, F., Botnen, A. \& ShalchianTabrizi, K.B.B. 2009: AIR: A batch-oriented web program package for construction of supermatrices ready for phylogenomic analyses. - BMC Bioinformatics 10 : 357.

Kytövuori, I., Niskanen, T., Liimatainen, K. \& Lindström, H. 2005: Cortinarius sordidemaculatus and two new related species, C. anisatus and C. neofurvolaesus, in Fennoscandia (Basidiomycota, Agaricales). - Karstenia 45: 33-49.

Kårén, O., Högberg, N., Dahlberg, A., Grip, K. \& Nylund, J.-E. 1996: Influence of drought on ectomycorrhizal species composition-morphotype versus PCR composition. - In: Azcon-Aguilar, C. \& Barea, J.M. (eds). My- 
corrhizas in integrated systems - from genes to plant development, 43-46. European Commission, Brussels.

Larson, R.T., Lorch, J.M., Pridgeon, J.W., Becnel, J.J., Clark, G.G. \& Lan, Q. 2010: The Biological Activity of $\alpha$-Mangostin, a Larvicidal Botanic Mosquito Sterol Carrier Protein-2 Inhibitor. - Journal of Medical Entomology 47: 249-257.

Lindström, H., Bendiksen, E., Bendiksen, K. \& Larsson, E. 2008: Studies of the Cortinarius saniosus (Fr.: Fr.) Fr. complex and a new closely related species, C. aureovelatus (Basidiomycota, Agaricales). - Sommerfeltia 31: 139-159.

Liu, Y., Rogers, S.O., Ammirati, J.F. \& Keller, G. 1995: Dermocybe, section Sanguineae. A look at species relationships within the sanguinea complex. - Beihefte zur Sydowia 10: 142-154.

Liu, Y., Rogers, S.O. \& Ammirati, J.F. 1997: Phylogenetic relationships in Dermocybe and related Cortinarius taxa based on nuclear ribosomal DNA internal transcribed spacers. - Canadian Journal of Botany 75: 519-532.

Maddison, W. \& Maddison, D. 1992: MacClade version 3: Analysis of phylogeny and character evolution. Sinauer Associates, Inc: Sunderland, Massachusetts.

Michael, L., Humphrey, J.S. \& Decker, D.G. 1997: Feeding Deterrence of Anthraquinone, Anthracene, and Anthrone to Rice-Eating Birds. - Journal of Wildlife Management 61: 1359-1365.

Moser, M. 1969: Cortinarius Fr., Untergattung Leprocybe subgen. nov., die Rauhköpfe. Vorstudien zu einer Monographie. - Zeitschrift für Pilzkunde 35: 213-248.

Moser, M. 1983: Die Röhrlinge und Blätterpilze (Polyporales, Boletales, Agaricales, Russulales). Gustav Fischer Verlag. Stuttgart.

Moser, M. \& Horak, E. 1975: Cortinarius Fr. und nahe verwandte Gattungen in Südamerika. - Beiheft zur Nova Hedwigia 52: 1-628.

Murray, M.G. \& Thompson, W.F. 1980: Rapid isolation of high molecular weight plant DNA. - Nucleic Acids Research 8: 4321-4326.

Niskanen, T. 2008. Cortinarius subgenus Telamonia p.p. in North Europe, Department of Biological and Environmental Sciences and Finnish Museum of Natural History. University of Helsinki, Finland, Helsinki, p. 33.

Niskanen, T., Kytövuori, I., Bendiksen, E., Bendiksen, K., Brandrud, T.E., Frøslev, T.G., Høiland, K., Jeppesen, T.S., Liimatainen, K. \& Lindström, H. 2008: Cortinarius (Pers.) Gray. - In: Knudsen, H. \& Vesterholt, J. (eds). Funga Nordica, 661-777. Nordsvamp. Copenhagen.

Niskanen, T., Kytövuori, I. \& Liimatainen, K. 2009: Cortinarius sect. Brunnei (Basidiomycota, Agaricales) in North Europe. - Mycological Research 113: 182-206.

Niskanen, T., Kytövuori, I. \& Liimatainen, K. 2011: Cortinarius sect. Armillati in northern Europe. - Mycologia 103: 1080-1101.

Niskanen, T., Laine, S., Liimatainen, K. \& Kytövuori, I. 2012: Cortinarius sanguineus and equally red species in Europe with an emphasis on northern European material. - Mycologia 104: 242-253.

Niskanen, T., Kytövuori, I., Liimatainen, K. \& Lindström, H. 2013a: The species of Cortinarius, section Bovini, asscocated with conifers in northern Europe. - Mycologia 105: 977-993.
Niskanen, T., Liimatainen, K., Ammirati, J.F. \& Hughes, K. 2013b: Cortinarius section Sanguinei in North America. - Mycologia 105: 344-356.

von Nussbaum, F., Spiteller, P., Rüth, M., Steglich, W., Wanner, G., Gamblin, B., Stievano, L. \& Wagner, F.E. 1998: An Iron(III) - Catechol Complex as a Mushroom Pigment. - Angewandte Chemie (International ed. in English) 37: 3292-3295.

Orton, P.D. 1958: The Genus Cortinarius II. Inoloma and Dermocybe. - Naturalist 1958: 81-149.

Peintner, U. 2008: Cortinarius alpinus as an example for morphological and phylogenetic species concepts in ectomycorrhizal fungi. - Sommerfeltia 31: 161-177.

Peintner, U., Bougher, N.L., Castellano, M.A., Moncalvo, J.-M., Moser, M.M., Trappe, J.M. \& Vilgalys, R. 2001; Multiple origins of sequestrate fungi related to Cortinarius (Cortinariaceae). - American Journal of Botany 88: $2168-2179$

Peintner, U., Horak, E., Moser, M.M. \& Vilgalys, R. 2002: Phylogeny of Rozites, Cuphocybe and Rapacea inferred from ITS and LSU rDNA sequences. - Mycologia 94: 620-629.

Peintner, U., Moser, M.M., Thomas, A.G. \& Manimohan, P. 2003: First records of ectomycorrhizal Cortinarius species (Agaricales, Basidiomycetes) from tropical India and their phylogenetic position based on rDNA ITS sequences. - Mycological Research 107: 485-494.

Peintner, U., Moncalvo, J.-M. \& Vilgalys, R. 2004: Toward a better understanding of the infrageneric relationships in Cortinarius (Agaricales, Basidiomycota). - Mycologia 96: 1042-1058.

Posada, D. \& Crandall, K.A. 1998: MODELTEST: testing the model of DNA substitution. - Bioinformatics 14: 817-818.

Ronquist, F. \& Huelsenbeck, J.P. 2003: MrBayes 3: Bayesian phylogenetic inference under mixed models. - Bioinformatics 19: 1572-1574.

Rudi, K., Kroken, M., Dahlberg, O.J., Deggerdal, A., Jakobsen, K.S. \& Larsen, F. 1997: Rapid, universal method to isolate PCR-ready DNA using magnetic beads. BioTechniques 22: 506-511.

Ryberg, M. \& Matheny, P.B. 2011: Asynchronous origins of ectomycorrhizal clades of Agaricales. - Proceedings of the Royal Society B: Biological Sciences: 9. doi:10.1098/rspb.2011.2428.

Schumacher, T. \& Høiland, K. 1983: Mushroom poisoning caused by species of the genus Cortinarius Fries. - Archives of Toxicology 53: 87-106.

Seidl, M.T. 2000: Phylogenetic relationships within Cortinarius subgenus Myxacium, sections Defibulati and Myxacium. - Mycologia 92: 1091-1102.

Singer, R. 1986: The Agaricales in Modern Taxonomy, ed 4. Koeltz Scientific Books. Koenigstein.

Singer, R. \& Smith, A.H. 1946: Proposals concerning the nomenclature of the gill fungi including a list of proposed lectotypes and genera conservanda. - Mycologia 38: $240-299$.

Soop, K. 2013: A contribution to the study of the cortinarioid mycoflora of New Zealand, V. - Australasian Mycologist 31: 1-9.

Spiteller, P. 2008: Chemical Defence Strategies of Higher Fungi. - Chemistry - A European Journal 14: 9100 9110. 
Spiteller, P., Rüth, M., von Nussbaum, F. \& Steglich, W. 2000: Detection of a 2,3-Aminomutase in the Mushroom Cortinarius violaceus. - Angewandte Chemie (International ed. in English) 39: 2754-2756.

Stamatakis, A. 2006: RAxML-VI-HPC: Maximum likelihood-based phylogenetic analyses with thousands of taxa and mixed models. - Bioinformatics 22: 26882690.

Stefani, F.O.P., Jones, R.H. \& May, T.W. 2014: Concordance of seven gene genealogies compared to phenotypic data reveals multiple cryptic species in Australian dermocyboid Cortinarius (Agaricales). - Molecular Phylogenetics and Evolution 71: 249-260.

Steglich, W., Steffan, B., Eizenhöfer, T., Fugmann, B., Herrmann, R. \& Klamann, J.-D. 1990: Some problems in the structural elucidation of fungal metabolites. - In: Chadwick, D.J. \& J., M. (eds). Bioactive compounds from plants, 56-65. John Wiley \& Sons Ltd. Chichester.

Suáres-Santiago, V.N., A., O., Peintner, U. \& Lópes-Flores, I. 2009: Study on Cortinarius subgenus Telamonia section Hydrocybe in Europe, with especial emphasis on Mediterranean taxa. - Mycological Research 113: 1070-1090.

Søli, G. \& Kjærandsen, J. 2008: Additions to the Norwegian fauna of fungus gnats (Diptera, Mycetophilidae). - Norwegian Journal of Entomology 55: 31-41.

Teichert, A., Schmidt, J., Porzel, A., Arnold, N. \& Wessjohann, L. 2008: (Iso)-Quinoline Alkaloids from Fungal Fruiting Bodies of Cortinarius subtortus. - Journal of Natural Products 71: 1092-1094.

Trial, H., Jr. \& Dimond, J.B. 1979: Emodin in buckthorn: a feeding deterrent to phytophagous insects. - Canadian Entomologist 111: 207-212.

White, T.J., T., B., Lee, S. \& Taylor, J. 1990: Amplification and direct sequencing of fungal ribosomal RNA genes for phylogenetics. - In: Michael, A.I., Gelfand, D.H., Sninsky, J.J. \& White, T.J. (eds). PCR Protocols: a Guide to Methods and Applications, 315-322. Academic Press. Inc. New York. 
